\title{
Posterior reversible encephalopathy syndrome in a child with unilateral renal artery stenosis with hypertensive emergency
}

\author{
Devdeep Mukherjee ${ }^{1}$, Prabhas Prasun Giri ${ }^{2}$, Priyankar Pal ${ }^{3}$, Rajiv Sinha $^{4}$, Agnisekhar Saha \\ Sri Lanka Journal of Child Health, 2014; 43(3): 168-170
}

(Key words: Posterior reversible encephalopathy syndrome; PRES; renal artery stenosis; hypertension)

\section{Case report}

A 7 year old boy was admitted with a history of fever for 10 days, intermittent convulsions, vomiting for 6 days and obtundation of sensorium for 3 days. Developmental history was appropriate for age and there was no past history or family history of seizures. There was no history of hypertension.

Blood counts done prior to admission showed a total leukocyte count (TLC) of 22,200/cu mm ( $82 \%$ neutrophils) and a C-reactive protein (CRP) of 24.64. Lumbar puncture (LP) showed 6 cells, all mononuclear, with normal protein and sugar levels. No organisms were isolated from blood or cerebrospinal fluid (CSF).

On admission, his pupils were dilated and reacting sluggishly to light. There was no evidence of papilloedema. He had brisk deep tendon reflexes and an extensor plantar response. He had a heart rate of 124/minute and a blood pressure (BP) of 242/126 mm Hg. He was initially managed with intravenous (IV) fluids, antibiotics and anticonvulsants (phenytoin) followed by continuous IV infusion of labetalol for the control of his hypertensive emergency.

Blood counts on admission showed that the TLC had normalised. CRP had come down to a normal range. Chest x-ray showed evidence of cardiomegaly. Echocardiography revealed left ventricular hypertrophy. However, as encephalopathy persisted, we did a magnetic

$\overline{1}$ Postgraduate Trainee, ${ }^{2}$ Resident Medical Officer,

${ }^{3}$ Assistant Professor, Department of Paediatric Medicine, ${ }^{4}$ Department of Paediatric Nephrology,

${ }^{5}$ Department of Paediatric Critical Care, Institute of Child Health, Kolkata, West Bengal, India

(Received on 26 May 2013: Accepted after revision on 26 July 2013) resonance imaging (MRI) of brain which was suggestive of vasculitis of bilateral parieto-occipital and frontal region possibly due to PRES (Figure 1).

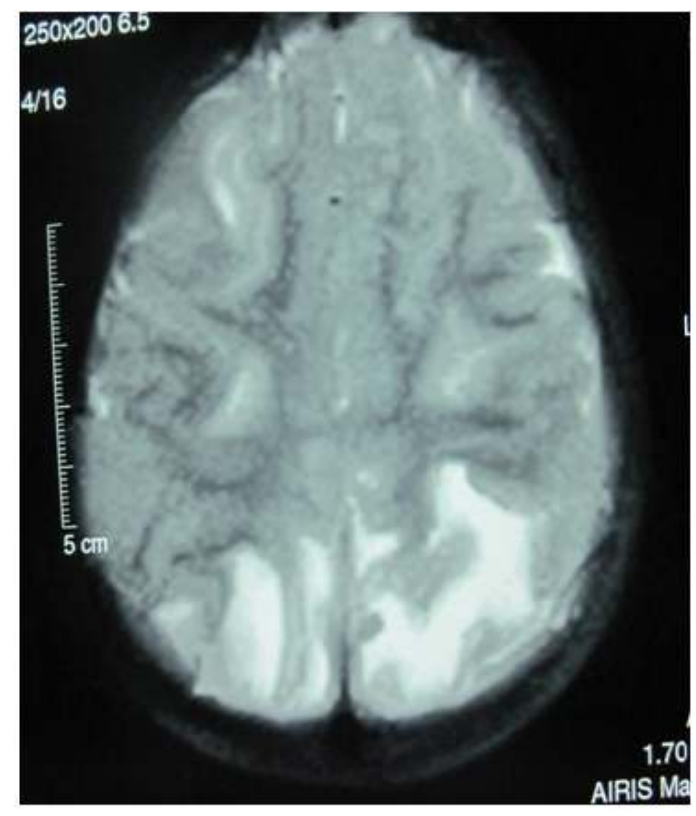

Figure 1: MRI of brain: suggestive of oedema of $B / L$ parieto-occipital region

Ultrasonography (USG) of abdomen with Doppler suggested asymmetry in kidney size and blood flow in both renal arteries. Subsequently, a computed tomography (CT) scan of abdomen with angiography of renal vessels was done which showed occlusion of left renal artery just distal to the origin, along with duplication of the artery. He had a small smooth left kidney with hypertrophy of right kidney (Figure 2). 

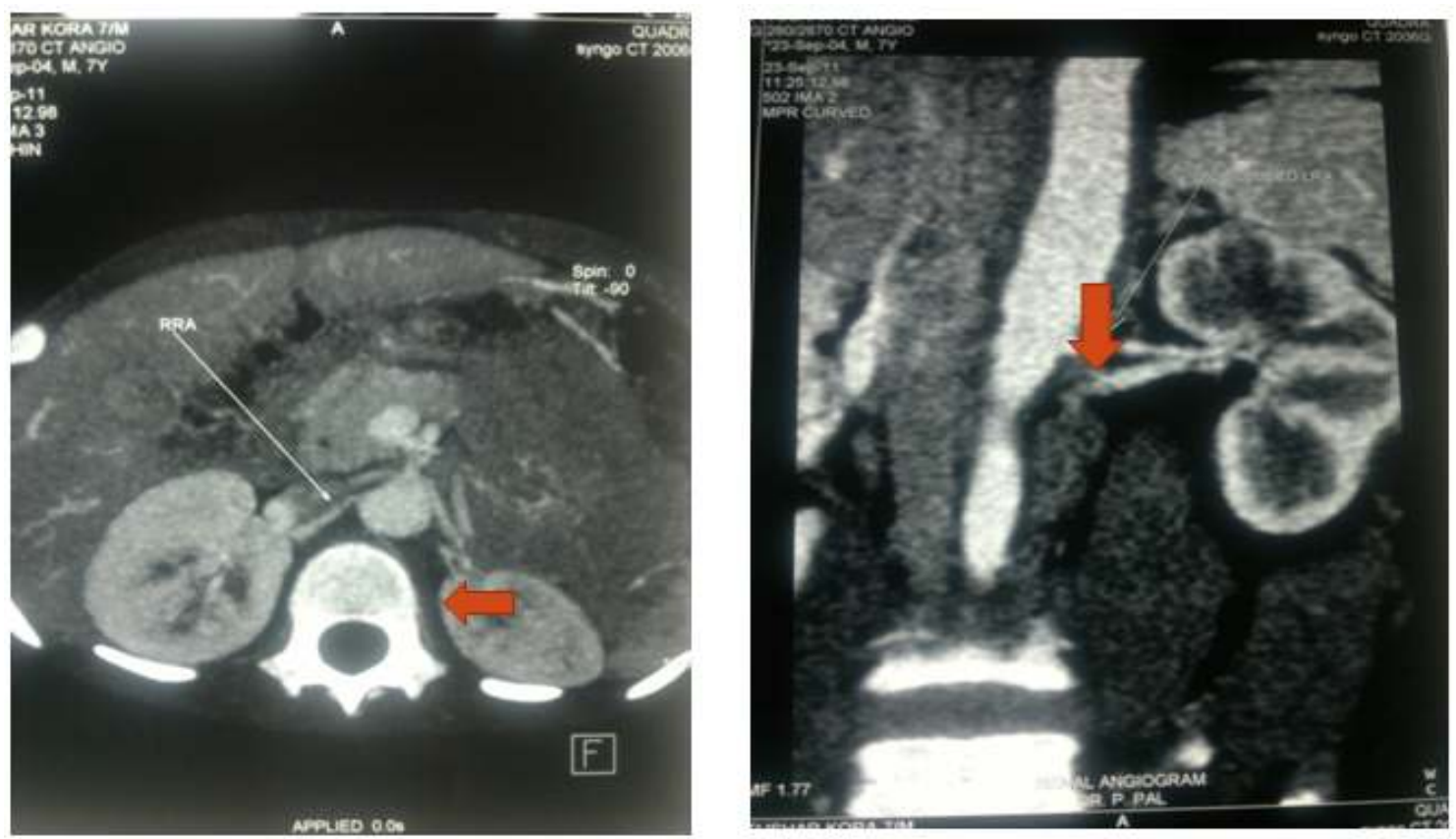

Figure 2: CT scan abdomen showing occlusion of left renal artery just distal to origin, small smooth left kidney with compensatory hypertrophy of right kidney and duplication of left renal artery

Control of hypertension and relevant investigations were carried out simultaneously. The hypertensive emergency was treated with labetalol infusion followed by oral amlodipine, enalapril and hydralazine. Gradually his BP came down to normal levels appropriate for age and sex.

Diethylene triamine pentaacetic acid (DTPA) scan was done after 2 weeks and showed the glomerular filtration rate (GFR) of the right kidney to be $82.17 \%$ and of left kidney to be $17.83 \%$.

The patient has presently been discharged on oral anti hypertensives (amlodipine and enalapril) till surgical intervention is availed of by them.

\section{Discussion}

Posterior reversible encephalopathy syndrome (PRES) is a neuro-radiologic condition associated with headache, nausea, vomiting, seizures, visual disturbances and transient motor deficits ${ }^{1}$. It is extremely rare in children. It may occur in diverse situations like hypertension, eclampsia, immunosuppressive (cyclosporine, methotrexate) therapy, renal failure and sepsis. In patients with PRES, seizures are common at the onset but can also develop later. The seizures are usually generalized tonic clonic in type. Temporary restlessness and agitation may alternate with lethargy. Our patient presented with predominant generalized convulsions, brisk deep tendon reflexes and extensor plantar response. He also had an obtunded sensorium.

There is predilection for white matter involvement. Like the index case, the parieto-occipital region of the brain is more commonly involved probably leading Hinchey to suggest the "posterior" description ${ }^{2}$. Other sites of involvement include the frontal lobe, temporal lobe and cerebellum ${ }^{3}$.

The underlying pathophysiology of PRES remains elusive. The most widely accepted theory states that rapidly developing hypertension leads to breakdown of cerebral autoregulation, particularly in the posterior region. Hypoperfusion ensues with protein and fluid extravasation, producing focal vasogenic oedema. An alternative theory mentions endothelial dysfunction. A third theory proposes that vasospasm with subsequent ischaemia may be responsible ${ }^{4,5}$.

PRES is potentially reversible. Appropriate therapy results in complete resolution over days to weeks. However, partial resolution has been reported and the disease can be fatal. Treatment of arterial blood pressure with anti-hypertensives, control of seizures with anticonvulsants and removing any offending drug, if any, is the treatment of choice ${ }^{6}$.

Hypertensive encephalopathy (an example of hypertensive emergency) consists of a combination of symptoms and signs such as nausea, vomiting, headaches, altered mental status, visual disturbances and seizures as we find in our 
patient. The treatment of hypertensive emergency should be started promptly, keeping in mind the clinical status of the patient, the aetiology, change in cardiac output or total peripheral resistance and whether there is end-organ involvement. Rapid reduction in blood pressure can interfere with adequate organ perfusion. Hence a stepwise reduction should be planned. Hypertensive emergencies should be treated by an IV antihypertensive that can produce a controlled reduction in the $\mathrm{BP}$, aiming to decrease the pressure by $25 \%$ over the first 8 hours after presentation and then gradually normalizing the BP over the next 48 hours $^{7}$. IV labetalol and sodium nitroprusside are the preferred agents. We managed the hypertensive emergency with labetalol. Subsequent management of BP was done with oral amlodipine, enalapril and hydralazine. We also followed the protocol of stepwise reduction of BP.

Unilateral renal artery stenosis as a cause of renovascular hypertension causing hypertensive emergency in children is uncommon. However it is a treatable condition when diagnosed early. Fibromuscular dysplasia is the most important cause in children. Correction of BP with anti hypertensives along with appropriate surgical interventions like balloon angioplasty or stent placement is the management of choice. However stent placement has not gained consensus in the paediatric population ${ }^{9}$. Our child responded well to the conventional anti-hypertensives.

The combination of unilateral renal artery stenosis with malignant hypertension leading to CNS manifestation like PRES is really a rare coexistence in the paediatric population.

\section{References}

1. Bartynski WS. Posterior reversible encephalopathy syndrome, Part 1: Fundamental imaging and clinical features American Journal of Neuroradiology 2008; 29: 1036-42. http://dx.doi.org/10.3174/ajnr.A0929
2. Hinchey J, Chaves C, Appignani B.et al. A reversible posterior leukoencephalopathy syndrome. New England Journal of Medicine 1996; 334: 494-500. http://dx.doi.org/10.1056/NEJM199602223340 $\underline{803}$

3. Fugate JE, Claassen DO, Cloft HJ, Kallmes DF, Kozak OS,Rabinstein AA. Posterior reversible encephalopathy syndrome: associated clinical and radiologic findings. Mayo Clinic Proceedings 2010; 85:427-32. http://dx.doi.org/10.4065/mcp.2009.0590

4. Bartynski WS. Posterior reversible encephalopathy syndrome, Part 2: Controversies surrounding pathophysiology of vasogenic edema. American Journal of Neuroradiology 2008; 29:1043-9. http://dx.doi.org/10.3174/ajnr.A0929

5. McKinney AM, Short J, Truwit CL, et al. Posterior reversible encephalopathy syndrome: incidence of atypical regions of involvement and imaging findings. American Journal of Roentgenology 2007; 189::904-12. http://dx.doi.org/10.2214/AJR.07.2024

6. Koshy AG. Acute hypertension and hypertensive crisis in children. Available from: http://www.pedheartsat.org/articles/Acute $\% 20$ Hypertension \%20and\%20Hypertensive \%20Cri sis $\% 20 \mathrm{in} \% 20$ Children.html

7. Bayazit AK, Yalcinkaya F, Cakar N, Duzova A, Bircan Z, Bakkaloglu A, et al. Renovascular hypertension in childhood: a nationwide survey. Pediatric Nephrology 2007; 22: 1327-33. http://dx.doi.org/10.1007/s00467-007-0520-4 